\title{
The latest development of Micro-nano manufacturing of next generation lithography technology
}

\author{
Zhongming Zhang ${ }^{a}$, Hongxue Wang ${ }^{b}$ \\ School of Management, Tianjin University of Technology, Tianjin 300384, China \\ a2384581998@qq.com, b842663678@qq.com
}

Keywords: Nano-fabrication, Technology node, EUV, NIL, DSA.

\begin{abstract}
This paper combined with the latest lithography technology development in recent years, and introduced the current mainstream technology of $32 \mathrm{~nm}$ technology node. Then focus on introducing the concept, technical characteristics, development situation and facing the challenge of next generation lithography technology candidates that extreme ultraviolet carved (EUV), electron beam direct writing lithography, nano-imprinting lithography (NIL) and directional self-assembly (DSA) technologies.
\end{abstract}

\section{Introduction}

In the manufacture of semiconductor integrated circuit, lithography technology consumes the longest time, accounts for the highest cost,40\% of the whole chip manufacturing cost. Photolithography line width is the important index of integrated circuit manufacturing. The development of lithography can determine whether the integrated circuit develop according to Moore's law.

Since 2004 , we can see that HP (half - pitch) of MPU and DRAM in 2003 has entered the $90 \mathrm{~nm}$ node, The requirements of FLASH are more strict. FLASH has reached $28 \mathrm{~nm}$ in 2013[1].By the year 2026 it is expected to reach $63 \mathrm{~nm}$, close to the limit of the integrated circuit manufacturing. When entering the micro-nano era, the traditional optical lithography technology can not meet the demand farly, even if he improved optical lithography. Because of the limitation of the limit ,the numerical aperture and process factor $\mathrm{K} 1$ in $22 \mathrm{~nm}$ node has not arrived. So the lithography giant refuses to give up the current skilled lithography, as the same time they are further developing new lithography technology and improving from the resolution, the respect such as cost, efficiency, so as to gain a foothold in the micro-nano competition. The extreme ultraviolet carved, electron beam direct writing photolithography, nano-imprinting lithography (NIL) and directional self-assembly (DSA) have become the most promising technology of next generation lithography technology[2].

\section{The present development situation of current mainstream lithography technology}

Excimer lithography. In the era of micro-nano, excimer lithography technology still is the mainstream technology, such as 0.1 um node 248 NMKRF excimer laser technology, 90 of the 90 nm node nmarf excimer laser technology, 65 nm node nmarf immersion lithography, of which 193 nmarf immersion lithography is the most competitive and has the most longevity.

193 nmarf immersion lithography in lithography projection replace air by the last of the objective lens surface between the photoresist on the silicon wafer with high refractive index of liquid, therefore under the same Angle of incidence, equivalent numerical aperture, process factor K1 all increase the $\mathrm{n}$ times ( $\mathrm{n}$ for refractive index), and then improve the resolution. $193 \mathrm{nmarf}$ immersion lithography technology does not need to develop a new mask, lens and resist material. Most of the components can keep dry lithography machine. Immersion exposure demands strictly for immersion liquid, we must consider the deposition of the water soluble materials, bubble, light absorbed at a constant temperature, illumination of the polarized light, and at the same time, nondestructive polarized light lighting system needs to be designed. Now it is widely used to ion and the pure water as immersion liquid. 
Double image exposure technique

Double exposure technique is the effective way to improve the resolution by extending to the 32 nm node. The principle is that a set of high density circuit graphics is decomposed into two separate low density of some graphics and then print them on the wafer.

The mainstream of the dual graphics technology solutions are:

1) lithography etching, lithography, etching (LELE) . First lithography etching hard mask process again on the second photolithography, and then the second photoresist and hard mask for the first time as a second etching mask, the whole process leave lithography once.

2) lithography - process - lithography - etching (LPLE) . First printing of the photoresist "freeze" in the second exposure that does not occur in the reaction, on the basis of the first photoresist coating the second photoresist. Finally ,completing the graph of two lithography etching process, the whole process does not leave the lithography[3].

High refractive index medium of immersion lithography

For manufacturers, they are trying to extend 193 nmarf immersion lithography technology continuously. High refractive index medium lithography extends to the $22 \mathrm{~nm}$ node by studying the high refractive index lens and high refractive index material of immersion fluid immersion technology.

In respect of immersion fluid material, the use of aqueous solution containing nanoparticles is the realization of the third generation of immersion fluid material, which is the optimal choice. Now we can get nanoparticles in aqueous solution of volume ratio of $10 \%$. Research teams are faced with the problem how to turn the volume ratio to increase to $37 \%$, and reduce the contamination of the residual organic matter in the process of synthesis, and improve the nanoparticles monomer dispersion and decrease the diameter of the nanoparticles in the making[4].

\section{The next generation lithography (NGL)}

When entering below the $22 \mathrm{~nm}$ node, the traditional 193 - nm immersion cannot be satisfied, even though Intel, IBM have the optimisticl attitude to its extension below the $22 \mathrm{~nm}$. but many big manufacturers have already began to enter into the next generation lithography. Carved with extreme ultraviolet (EUV), electron beam direct writing lithography, nano-imprinting lithography (NIL) and directional self-assembly (DSA) are four of the most potential candidate technologies.

Extreme ultraviolet lithography

The traditional way of improving the resolution is to increase the numerical aperture (NA) or shorten the exposure wavelength. EUV (extreme ultraviolet lithography) is the next generation lithography technology $(<32 \mathrm{~nm}$ lithography). It is with the method of shorting wavelength to improve resolution, usually adopting very short wavelength far ultraviolet light of 11-14 nm . Compared to conventional optical lithography, it absorbs the material that is under extremely short wavelength light seriously, so the EUV is based on the total reflection optical projection, using reflection mask. The development speed of EUV lithography is slow for 193 - nm immersion lithography technology extension.

The challenge for the current EUV lithography are:

1)Research high power project (greater than $115 \mathrm{w}$ ), the light source system with high rate and high life; Existing laser power in extreme ultraviolet is small, short of the energy needed to power a lithography and expensive. High power laser source system development is the key to reduce the holistic machine cost.

2) Manufactue reflection lens and reflection mask that meet nanoscale lithography, no fatal flaw, the structure of the multilayer reflection film.

Electron beam direct writing photolithography

Electron beam lithography has become a high-profile technology in the micro-nano era for its high resolution, stable performance, powerful function, the characteristics of the price is relatively low. Electron beam lithography has direct writing type and projection type two kinds. Direct writing type is directly to the convergence of electron beam spot on the surface coated with photoresist substrate, 
and do not need to be the most expensive in optical lithography process and preparation of time-consuming mask. As no mask technique has been ITRS into the $22 \mathrm{~nm}$ node alternative, direct write lithography have more advantages in the next generation lithography technology.

The wavelength of the electron beam lithography depends on the electron beam energy, the higher the energy the shorter the wavelength, generally in $10 \wedge 6 \mathrm{~nm}$ wavelength. As electron diffraction cannot affect the resolution . Electron beam near effect is seriously affecting the resolution. The high energy electron beam irradiation on the photoresist many electrons which resulted from the small angle scattering of light, when the electron beam into the substrate further some electronic produce large angle scattering (back scattering) planned unexposed electronics into the area, make originally not exposure area expose. Electron beam near effect causes excessive exposure, which lead to graphics deformation[5].

Nano-imprinting technologies (NIL) and directed self-assembly technology (DSA). Compared with the known EUV and electron beam direct writing, NIL and DSA is the advanced lithography technology, which emerged in recent years despite still in the air to achieving these two technologies and related report is less poor, but its technology advancement let I had to introduce the two innovative lithography technology. I believe that when close to limit the photolithography process, the two technologies are more competitive.

Nano-imprinting technologies (NIL). The study of nano-imprinting lithography technology starts from nanostructures laboratory in Princeton University. Professor Stephen Y.Chou has a design template to mechanical force (high temperature and high pressure) in silicon substrate coated with polymer material proportion of fine embossing replication nano pattern, its processing resolution is only related to the size of the template, and there is no optical lithography exposure of the shortest wavelength of physical restrictions. It can be large quantities of repetitive in large area on the preparation of nanometer structure of graphics, and produced by the high resolution design has fairly good uniformity and repeatability. Due to eliminating optical lithography mask template and the cost of using optical imaging equipment, NIL technology has low cost, highly productive economic advantage. The most advanced new 193 - nm immersion scanning lithography equipment cost \$25 million to $\$ 40$ million, nano-imprinting equipment sells for $\$ 100000$ or so, the cost advantage is very obvious[6].

Directed self-assembly technology (DSA). NIL is lithography technology innovation, the DSA have made a new breakthrough with NIL technology in principle. Compared with DSA in terms of the above three kinds of technology, few people know, let alone understand, and the relevant report is also very little, but it is a technology that cannot be ignored. DSA technology deposites block polymer or polymer mixture on the substrate, usually by spin coating, and through the annealing process with 'command'the orderly structure. The researchers point out that DSA is compatible with traditional 193 - nm lithography equipment, no longer need to double exposure step.

European research institutions in 2012 directed self-assembly (DSA) process, allegedly can improve optical and super uv lithography, and has set up a pilot installing in the line of $300 \mathrm{~mm}$. TEL and imec company's latest assessment DSA technology in 2012 realize the frequency doubling of fine graphics by using the accumulation copolymer,. Imec $300 \mathrm{~mm}$ wafer oriented apex lithography combined with DSA technology, formation of $12.5 \mathrm{~nm}$ bar is the graphics and $25 \mathrm{~nm}$.

\section{Summary}

This article overviewed the development of lithography in nearly three years. In $32 \mathrm{~nm}$ node, immersion lithography is still in a leadership position, and has been mature, but the $22 \mathrm{~nm}$ node is not good obviously. Four kinds of next generation lithography technology have their own advantages, and they face big challenges. Any a technology breakthrough will lead the new wave under $22 \mathrm{~nm}$. But I believe that the combination of technology according to different technical requirements can accelerate the development of lithography process. Before $10 \mathrm{~nm}$, it still gives priority to with immersion and ultraviolet light etching technology, secondary electron beam direct writing lithography. After $10 \mathrm{~nm}$ node, however, NIL and DSA will have much advantage. 


\section{References}

[1] Zhang Kun. The principle of Electron beam lithography technology and its application in micro-nano processing and the application of nano-electronic devices [J]. Journal of Electron Microscopy. Journal of Chinese Electron Microscopy Society. 2006:98-103

[2] Lithography_12052012itrs [R]. ITRS international semiconductor technology blueprint report Lithography.

[3] Photolithography technology: the trend of development path and future [J]. Journal of image and optical science. 2012, 1:1-8

[4] Jian-jun Ma 1,Chong-wei Zhang :keys to $22 \mathrm{~nm}$ immersion lithography [J]. Journal of electronic industrial specialized equipment. 2009 8) : 1-8 + 50

[5] Yao xin tree. Based on the $32 \mathrm{~nm}$ lithography dual graphics technology research and practice [D]. Shanghai: fudan university, 2011

[6] Wei-guo Liu. EUV light [J]. Science and technology progress of science and technology information. Science\&technology information. 2011, 21:44-418

[7] EUV equipment price of \$120 million [J]. Journal of electronics industry. 2010, 11:61 